\title{
Capture of dark matter by the Solar System. Simple estimates
}

\author{
I.B. Khriplovich 1 \\ Budker Institute of Nuclear Physics \\ 630090 Novosibirsk, Russia,
}

\begin{abstract}
We consider the capture of galactic dark matter by the Solar System, due to the gravitational three-body interaction of the Sun, a planet, and a dark matter particle. Simple estimates are presented for the capture cross-section, as well as for density and velocity distribution of captured dark matter particles close to the Earth.
\end{abstract}

\section{Introduction}

The density of dark matter $(\mathrm{dm})$ in our Galaxy is (see, e.g., [1])

$$
\rho_{g} \simeq 4 \cdot 10^{-25} \mathrm{~g} / \mathrm{cm}^{3} .
$$

However, only upper limits on the level of $10^{-19} \mathrm{~g} / \mathrm{cm}^{3}$ (see [2, 3]) are known for the density of dark matter particles (dmp) in the Solar System (SS). Besides, even these limits are derived under the quite strong assumption that the distribution of dm density in the SS is sphericallysymmetric with respect to the Sun. Meanwhile, information on dm density in SS is very important, in particular for the experiments aimed at the detection of dark matter.

The capture of dark matter by the SS was addressed previously in $[4-8]$. In particular, in [8] the total mass of the captured dark matter was estimated analytically. In the present note the analytical estimates are given for the capture cross-section, as well as for the density and velocity distribution of captured dm close to the Earth.

Of course, a particle cannot be captured by the Sun alone. The interaction with a planet is necessary for it, i.e. this is essentially a three-body (the Sun, planet and dmp) problem. Obviously, the capture is dominated by the particles with orbits close to parabolic ones with respect to the Sun; besides, the distances between their perihelia and the Sun should be comparable with the radius of the planet orbit $r_{p}$. Just the trajectories of these particles are most sensitive to the attractive perturbation by the planet.

The capture can be effectively described by the so-called restricted three-body problem (see, e.g., [9]). In this approach the interaction between two heavy bodies (the Sun and a planet in our case) is treated exactly. As exactly is treated the motion of the third, light body (a dmp in our case) in the gravitational field of the two heavy ones. One neglects however the

${ }^{1}$ khriplovich@inp.nsk.su 
back reaction of a light particle upon the motion of the two heavy bodies. Obviously, this approximation is fully legitimate for our purpose.

Still, the restricted three-body problem is rather complicated, its solution requires both subtle analytical treatment and serious numerical calculations (see, for instance, [10]). Under certain conditions the dynamics of light particle becomes chaotic. The "chaotic" effects are extremely important for the problem. However their quantitative investigation is quite complicated and remains beyond the scope of the present note. We confine here instead to simple estimates which could be also of a methodological interest by themselves. On the other hand, thus derived results for the total mass and density of the captured dark matter constitute at least an upper limit for their true value. As to the velocity distribution of dmp's given here, together with the mentioned result for the dark matter density, it could be possibly of some practical interest for planning the experimental searches for $\mathrm{dm}$.

\section{Total mass of dark matter captured by the Earth}

The Solar System is immersed in the halo of dark matter and moves together with it around the center of our Galaxy. To simplify the estimates, we assume that the Sun is at rest with respect to the halo. The dark matter particles in the halo are assumed also to have in the reference frame, comoving with the halo, the Maxwell distribution (see [1]]):

$$
f(v) d v=\sqrt{\frac{54}{\pi}} \frac{v^{2} d v}{u^{3}} \exp \left(-\frac{3}{2} \frac{v^{2}}{u^{2}}\right),
$$

with the local $\mathrm{rms}$ velocity $u \simeq 220 \mathrm{~km} / \mathrm{s}$. Let us note that the velocities $v$ discussed in this section are the asymptotic ones, they refer to large distances from the Sun, so that their values start at $v=0$ and formally extend to $\infty$.

The amount of dm captured by the SS can be found by means of simple estimates 2 . The total mass captured by the Sun (its mass is $M$ ) together with a planet with the mass $m_{p}$, during the lifetime

$$
T \simeq 4.5 \cdot 10^{9} \text { years } \simeq 10^{17} \mathrm{~s}
$$

of the SS, can be written as follows:

$$
\mu_{p}=\rho_{g} T<v \sigma>
$$

here $\sigma$ is the capture cross-section. The product $\sigma v$ is averaged over distribution (2); with all typical velocities in the SS much smaller than $u$, this distribution simplifies to

$$
f(v) d v=\sqrt{\frac{54}{\pi}} \frac{v^{2} d v}{u^{3}} .
$$

To estimate the average value $\langle v \sigma\rangle$, we resort to dimensional arguments, supplemented by two rather obvious physical requirements: the masses $m_{p}$ and $M$ of the two heavy components of our restricted three-body problem should enter the result symmetrically, and the mass of the dmp should not enter the result at all in virtue of the equivalence principle. Thus, we arrive at

$$
<v \sigma>\sim \sqrt{54 \pi} \frac{k^{2} m_{p} M}{u^{3}}
$$

\footnotetext{
${ }^{2}$ These estimates were given previously in [8]. Here we repeat them, as well as results (8), (9), (19) (see below), since they are essential for the present discussions.
} 
or

$$
\int_{0}^{\infty} d v v^{3} \sigma \sim \pi k^{2} m_{p} M
$$

here $k$ is the Newton gravitation constant; an extra power of $\pi$, inserted into these expressions, is perhaps inherent in $\sigma$. Since the capture would be impossible if the planet were not bound to the Sun, it is only natural that the result is proportional to the corresponding effective "coupling constant" $k m_{p} M$. One more power of $k$ corresponds to the gravitational interaction of the dark matter particle. The final estimate for the captured mass is

$$
\mu_{p} \sim \rho_{g} T \sqrt{54 \pi} k^{2} m_{p} M / u^{3}
$$

For the Earth it constitutes

$$
\mu_{E} \sim 4 \cdot 10^{18} \mathrm{~g}
$$

\section{Capture cross-section}

By the same dimensional reasons (and in the complete correspondence with formula (7)), the total capture cross-section for the Earth should look as follows:

$$
\sigma \sim \pi k^{2} m_{E} M / \tilde{v}^{4}
$$

where $m_{E}$ is the mass of the Earth, and $\tilde{v}$ is some velocity which can be estimated as follows. It is natural to assume that the capture of dm particles occurs when they are close to the Earth, i.e. at the distances $\sim r_{E}$ from the Sun. As natural are the following assumptions: 1) the initial velocities of the captured dmp's exceed only slightly the parabolic one $v_{\text {par }}\left(v_{\mathrm{par}}^{2}=2 k M / r_{E}\right)$; 2 ) their final velocities are only slightly less than $v_{p a r}$. To our accuracy, here we omit the factor of 2 in the definition of $v_{\text {par }}^{2}$, and thus put $\tilde{v}^{2} \sim v_{E}^{2}=k M / r_{E}\left(v_{E}=30 \mathrm{~km} / \mathrm{s}\right.$ is the velocity of the Earth). Thus, the capture cross-section is

$$
\sigma \sim \pi k^{2} m_{E} M / v_{E}^{4}
$$

This formula can be also conveniently rewritten as

$$
\sigma \sim \pi r_{E}^{2}\left(m_{E} / M\right)
$$

Let us note here that the impact parameter corresponding to formula (12), i.e. the typical distance between a dmp and the Earth crucial for the capture, is

$$
r_{\text {imp }} \sim r_{E}\left(m_{E} / M\right)^{1 / 2} \ll r_{E} .
$$

In fact, this impact parameter corresponds to the distance at which the attraction to the Earth equals the attraction to the Sun, i.e. where

$$
k m / r^{2}>k M / r_{E}^{2} \quad\left(r \ll r_{E}\right) .
$$

Up to now, in all relevant formulae, (17), (10), (11), we dealt with the capture cross-section averaged over the directions of the dmp velocity $\mathbf{v}$. However, this cross-section depends essentially on the mutual orientation of $\mathbf{v}$ and $\mathbf{v}_{E}$. Certainly, it is maximum when these velocities are parallel and as close as possible by modulus. Besides, the impact parameter $r_{\text {imp }}$ of the 
collision is much less than the radius $r_{E}$ of the Earth orbit (see (13)), and thus within the distances $\simeq r_{\mathrm{imp}}$ both the Earth and dmp trajectories can be treated as rectilinear. Therefore, it looks quite natural to identify $\tilde{v}$ in (10) with the relative velocity $\left|\mathbf{v}-\mathbf{v}_{E}\right|$ of the dmp and the Earth, i.e. to generalize formula (11) as follows:

$$
d \sigma \sim \frac{k^{2} m_{p} M}{\left(\mathbf{v}-\mathbf{v}_{E}\right)^{4}} \frac{1}{4} d \Omega
$$

(factor $1 / 4$ is introduced here for correspondence with factor $\pi$ in (11): $(1 / 4) \int d \Omega=\pi$ ).

Thus derived total cross-section is

$$
\sigma \sim \frac{1}{4} \int d \Omega \frac{k^{2} m_{p} M}{\left(\mathbf{v}-\mathbf{v}_{E}\right)^{4}}=\frac{\pi k^{2} m_{p} M}{\left(v^{2}-v_{E}^{2}\right)^{2}} .
$$

Clearly, it is the particles moving initially with the velocities only slightly above the parabolic one $\sqrt{2} v_{E}=42 \mathrm{~km} / \mathrm{s}$ that are captured predominantly, and thus, with $v=\sqrt{2} v_{E}$, cross-sections (11) and (16) practically coincide.

On the other hand, it follows from (16) that in the vicinity of the Earth the captured particles move with respect to it with the velocities close to $(\sqrt{2}-1) v_{p} \simeq 12 \mathrm{~km} / \mathrm{s}$.

\section{Space distribution of captured dark matter}

The captured dmp's had initial trajectories predominantly close to parabolas focussed at the Sun, and the velocities of these dmp's change only slightly as a result of scattering. Therefore, their trajectories become elongate ellipses with large semimajor axes, still focussed at the Sun. The ratio of their maximum $r_{\max }$ and minimum $r_{\min }$ distances from the Sun is [12]

$$
\frac{r_{\max }}{r_{\min }}=\frac{1+e}{1-e}
$$

where $e$ is the eccentricity of the trajectory. In our case, as a result of the capture, the eccentricity changes from $1+\varepsilon_{1}$ to $1-\varepsilon_{2}$, where $\varepsilon_{1,2} \ll 1$. This loss of eccentricity is due to the gravitational perturbation by the Earth, and therefore is proportional to $m_{E}$. On the other hand, $r_{\min }$ is close to the radius $r_{E}$ of the Earth orbit. Thus, for dimensional reasons, we arrive at 8 ]

$$
r_{\max } \sim r_{E}\left(M / m_{E}\right) .
$$

Let us note here that the analogous estimate for the case of Jupiter complies qualitatively with the results of corresponding numerical calculations presented in [10].

Obviously, the semimajor axis $a_{\mathrm{dmp}}$ of the trajectory of a captured dmp is on the same order of magnitude as $r_{\max }$. Then, the time spent by a dmp, with the characteristic velocity close to $v_{E}$ and at the distance from the Sun close to $r_{E}$, is comparable to the orbital period of the Earth $T_{E}=1$ year. Besides, the orbital period $T$ is related to the semimajor axis $a$ as follows [12: $T \sim a^{3 / 2}$. Thus, we arrive at the following estimate for the orbital period of the captured dmp:

$$
T_{\mathrm{dmp}} \sim T_{E}\left(M / m_{E}\right)^{3 / 2}
$$

In other words, the relative time spent by a dmp at the distances $\sim r_{E}$ from the Earth can be estimated as $\left(m_{E} / M\right)^{3 / 2}$. Moreover, the typical distances from the Earth at which a dmp

\footnotetext{
${ }^{3}$ In the case of the Earth, this orbital period is huge, $\sim 10^{8}$ years. Still, it is much less than the lifetime of the SS, $\sim 5 \cdot 10^{9}$ years.
} 
can be captured, should be less than the impact parameter $r_{\text {imp }} \sim r_{E}\left(m_{E} / M\right)^{1 / 2}$ (see (13)) . Thus, the relative time spent by a dmp sufficiently close to the Earth to be captured, can be estimated as $\left(m_{E} / M\right)^{2}$.

With the impact parameter (13), the corresponding volume $V$, centered at the Earth and crucial for the capture, can be estimated as

$$
V \sim \frac{4 \pi}{3} r_{\mathrm{imp}}^{3} \sim \frac{4 \pi}{3} r_{E}^{3}\left(m_{E} / M\right)^{3 / 2} \ll \frac{4 \pi}{3} r_{E}^{3} .
$$

Let us combine formula (9) for the total captured mass with the effective volume (20) occupied by this mass and with the estimate $\left(m_{E} / M\right)^{2}$ for the relative time spent by a dmp within the impact parameter (13) with respect to the Earth. In this way we arrive at the following estimate for the density of dark matter, captured by the SS, in the vicinity of the Earth:

$$
\rho_{E} \sim 5 \cdot 10^{-25} \mathrm{~g} / \mathrm{cm}^{3} .
$$

This estimate practically coincides with the value (1) for the galactic dm density.

In fact, the result (21) for the density of the captured dm, as well as the estimates (8) and (9) for its total mass, should be considered as upper limits only, since we have neglected therein the inverse process, that of the ejection of the captured dm from the SS. The characteristic time of the inverse process is not exactly clear now. Therefore, it cannot be excluded that it is comparable to, or even larger than, the lifetime $T$ of the SS [8]. In this case our estimates are valid.

If this is the case indeed, then the dm around the Earth consists essentially of two components with comparable densities. In line with the common component with the typical velocity around $u \sim 220 \mathrm{~km} / \mathrm{s}$, there is one more, with the velocity relative to the Earth $\gtrsim 12 \mathrm{~km} / \mathrm{s}$.

Acknowledgements. I am grateful to V.V. Sokolov for useful discussions. The work was supported by the Russian Foundation for Basic Research through Grant No. 08-02-00960-a.

\section{References}

[1] G. Bertone, D. Hooper, and J. Silk, Phys. Rep. 405, 279 (2005).

[2] I.B. Khriplovich and E.V. Pitjeva, Int. J. Mod. Phys. D 15, 615 (2006).

[3] I.B. Khriplovich, Int. J. Mod. Phys. D 16, 1475 (2007).

[4] A. Gould and S.M.K. Alam, Astrophys. J. 549, 72 (2001).

[5] J. Lundberg and J. Edsjo, Phys. Rev. D 69 (2004) 123505.

[6] A.H.G. Peter and S. Tremaine, Proc. of Sc. IDM 2008; arXiv:0806.2133 (astro-ph)

[7] A.H.G. Peter, Phys. Rev. D 79, 1003531, 1003532, 1003533 (2009).

[8] I.B. Khriplovich and D.L. Shepelyansky, Int. J. Mod. Phys. D 18, 1903 (2009).

[9] V. Szebehely, Theory of Orbits, Academic Press, N.Y. (1964).

[10] T.Y. Petrosky, Phys. Lett. A 117, 328 (1986).

[11] M.S. Alenazi and P. Gondolo, Phys. Rev. D 74, 083518 (2006).

[12] L.D. Landau and E.M. Lifshitz, Mechanics, Nauka, Moscow (1988), §15. 